\title{
Allopregnanolone in the brain and blood after disruption of the hypothalamic-pituitary-adrenal axis in fetal sheep
}

\author{
Phuong N Nguyen, I Ross Young, David W Walker and \\ Jonathan J Hirst
}

Fetal and Neonatal Research Group, Department of Physiology, Monash University, Clayton, Victoria 3800, Australia

(Requests for offprints should be addressed to J Hirst; Email: jon.hirst@med.monash.edu.au)

\begin{abstract}
Neuroactive steroids may be synthesised in the brain either de novo from cholesterol or from blood-borne precursors. Concentrations of a $\mathrm{GABA}_{\mathrm{A}}$ receptor agonist, allopregnanolone, in the fetal brain exceed those in the circulation, and are markedly higher than adult brain concentrations. We used fetal hypophysectomy or adrenalectomy to elucidate the contribution of hypothalamic-pituitary factors and adrenal steroid secretion to the overall neuroactive steroid level in both the fetal brain and the fetal circulation. Hypophysectomy or adrenalectomy was performed between 108 and 112 days of gestation (term $\sim 147$ days) and fetal tissues were collected at 140 days of gestation. Immunoreactive (ir) ACTH and cortisol in the plasma were significantly reduced after hypophysectomy, whereas adrenalectomy led to increased irACTH but significantly decreased cortisol concentrations, as expected. Brain concentrations of allopregnanolone, progesterone and pregnenolone did not change significantly in fetuses that underwent either hypophysectomy or adrenalectomy;
\end{abstract}

however, concentrations in the plasma and content in the adrenal gland were decreased. Expression of cytochrome P450 scc and $5 \alpha$-reductase type II ( $5 \alpha$ RII) in the brain, measured by western immunoblotting, did not change after either hypophysectomy or adrenalectomy but, after hypophysectomy, expression of P450 scc in the adrenal gland was significantly decreased and that of $5 \alpha \mathrm{RII}$ remained unchanged. These findings suggest that the regulation of the neuroactive steroid content in the fetal brain is independent of adrenal steroidogenesis and hypothalamic-pituitary factors. Furthermore, the absence of a change in enzyme expression in the brain suggests that the control of the expression of these enzymes is independent of hypothalamic-pituitary factors. Thus local control mechanisms within the brain may be responsible for maintaining the high neurosteroid content present during fetal life, as these mechanisms are independent of adrenal steroid production.

Journal of Endocrinology (2004) 182, 81-88

\section{Introduction}

Neurosteroids such as allopregnanolone have sedative properties that result from their stimulation of $\gamma$-aminobutyric acid $(\mathrm{GABA})_{\mathrm{A}}$ receptors, leading to increased GABAergic inhibition. Allopregnanolone binds to a specific site on the $G_{A B A}$ receptor to enhance GABAergic activity and has been shown to have a major role in regulating central nervous system (CNS) activity in the fetus, neonate and adult (Crawley et al. 1986, Nicol et al. 1998). Neurosteroids may be synthesised in the brain either de novo from cholesterol or from precursors derived from the circulation. Basal concentrations of allopregnanolone in the adult brain have been found to be independent of gonadal and adrenal sources, as concentrations were maintained after ablation or pharmacological suppression of the gland (Purdy et al. 1991, Cheney et al. 1995). Alternatively, neurosteroids and their precursors may be supplied from the circulation and are readily taken up across the blood-brain barrier. Pomata and colleagues (2000) showed that fetal rat brain and spinal cord produce neurosteroids from circulating progesterone by day 13 of pregnancy. These findings suggest that central and peripherally derived neurosteroids or their precursors may contribute to the regulation of neural function.

We have previously found that allopregnanolone concentrations are remarkably high in the ovine fetal brain from $0 \cdot 6$ of gestation ( 90 days, term 148 days), and that this neurosteroid has the effect of suppressing activity of the fetal CNS (Crossley et al. 2000, Nguyen et al. 2003a). We have also shown that acute fetal asphyxia increased the content of allopregnanolone in brain and adrenal tissues and in the plasma (Nguyen et al. 2003b). Furthermore, chronic fetal hypoxaemia caused by prolonged placental insufficiency resulted in increased plasma cortisol concentrations and upregulation of the expression of cytochrome P450 scc in the fetal brain (Nguyen et al. 2003c), a key rate-limiting step in the steroidogenic pathway. 
The mechanisms that regulate expression of neurosteroidogenic enzymes in the fetal brain have not been well investigated, but the increase in expression of some neurosteroidogenic enzymes in response to stress would be consistent with a role for glucocorticoids in the fetal circulation. The fetal adrenal gland possesses all the enzymes necessary for the synthesis of progesterone and its metabolism to $5 \alpha$-reduced neurosteroids, including allopregnanolone, and their precursors (Miller et al. 1987). These observations suggest that secretion of neurosteroid precursors by the fetal adrenal gland could contribute to the high concentrations in the fetal brain. In the adrenal gland, expression of P450 scc is under the control of adrenocorticotropic hormone (ACTH) and represents a major regulatory step in adrenal steroidogenesis (Simmonds et al. 2001). However, it is not clear whether the same mechanisms have a role in the regulation of $\mathrm{P} 450$ scc expression in the fetal brain.

During late gestation, allopregnanolone concentrations in the fetal brain are markedly greater than adult concentrations, and they increase further in response to stressors during pregnancy (Nguyen et al. 2003a, b, c). The production of allopregnanolone - and particularly its precursor, pregnenolone - by the fetal adrenal gland may have a major role in determining neurosteroid concentrations in the brain, with subsequent effects on fetal CNS activity (Nicol et al. 2001). The aim of this study was to determine the influence of the hypothalamopituitary-adrenal (HPA) axis on concentrations of allopregnanolone in the fetal brain, and to determine whether the expression of two key rate-limiting steroidogenic enzymes, P450 scc and $5 \alpha$-reductase type II ( $5 \alpha \mathrm{RII})$, in the fetal brain are influenced by hypothalamic factors or adrenal steroids. We used surgical hypophysectomy or adrenalectomy to examine the contribution of the fetal HPA axis to the regulation of allopregnanolone concentration and enzyme expression in the fetal brain. We found that, although these interactions markedly reduced plasma concentrations of allopregnanolone, there was no change in concentrations in the fetal brain. Our findings suggest that fetal brain and plasma neurosteroid concentrations are regulated by independent mechanisms.

\section{Materials and Methods}

All procedures were conducted in accordance with the Code of Practice for the Care and Use of Animals for Scientific Purposes of the National Health and Medical Research Council and had received prior approval from the Monash University Standing Committee on Ethics in Animal Experimentation. Border Leicester/Merino crossbred ewes, carrying either twin or singleton fetuses of known gestational age were used. The ewes were housed in individual cages under conditions of $12 \mathrm{~h}$ light: $12 \mathrm{~h}$ darkness and were fed once daily between $0900 \mathrm{~h}$ and
$1200 \mathrm{~h}$, with water available ad libitum. Surgery was performed between 108 and 112 days of gestation, using aseptic techniques. General anaesthesia was induced by intravenous injection of thiopentone sodium $(20 \mathrm{mg} / \mathrm{kg}$; Pentothal, Bomac Laboratories Ltd, Asquith, NSW, Australia) and maintained by inhalation of $1-2 \%$ halothane (Fluothane; ICI, Villawood, Australia) in oxygen.

\section{Hypophysectomy}

Six fetuses underwent hypophysectomy as described previously (Mesiano et al. 1987). Briefly, the uterus was exposed through a midline abdominal incision and the fetal head was exposed. A parapharyngeal incision was made and careful blunt dissection was performed to expose the fetal sphenoid bone. A small hole was drilled through the bone to expose the underlying pituitary gland. Using microscope visualisation, the gland was removed by aspiration. Hypophysectomy was considered complete when the entire pituitary fossa could be inspected. The fetus was then returned to the uterus and all maternal incisions were repaired. Completeness of hypophysectomy and lack of damage to the ventral surface of the brain were also confirmed at postmortem in all six fetuses.

\section{Adrenalectomy}

Six fetuses underwent bilateral adrenalectomy as described previously (Simonetta et al. 1993). Briefly, after the uterus had been exposed, the fetal lumbar area was located and an incision was made through the uterus, membranes and fetal skin. Bilateral paralumbar incisions were made for the retroperitoneal approach to the adrenal glands. The adrenal gland was mobilised by blunt dissection and blood vessels were coagulated and cut by bipolar diathermy; dissection continued until the gland was free and could be removed. After the removal of both adrenal glands, the fetal skin was sutured and the fetus was returned to the uterus.

\section{Intact fetuses}

An additional five animals underwent surgery in which the uterus was opened and the fetus was exposed, but the adrenal glands and pituitary glands were left intact.

\section{Tissue collection}

All ewes and fetuses were killed by injection of an overdose of sodium pentobarbitone $(130 \mathrm{mg} / \mathrm{kg}$ i.v.) given to the ewe at 140 days of gestation. A fetal blood sample was obtained before the animal was killed, and completeness of hypophysectomy or adrenalectomy was assessed by histological examination of the pituitary fossa and the retroperitoneal space, respectively. The fetal brain was removed and divided into blocks corresponding to 
the following regions: cerebellum, hippocampus, medulla, midbrain, pons, hypothalamus/thalamus, primary motor cortex (PMC) and frontal, parietal, occipital and temporal cortexes. Fetal adrenal glands were collected from sham-operated and hypophysectomised fetuses. The tissue was frozen in liquid nitrogen and stored at $-70{ }^{\circ} \mathrm{C}$.

\section{Immunoblotting}

Expression of cytochrome P450 scc and $5 \alpha \mathrm{RII}$ in the brain and adrenal glands was determined by Western immunoblotting as described previously (Nguyen et al. 2003a). In brief, frozen samples $(\sim 0.1 \mathrm{~g})$ from each brain or adrenal glands were homogenised and then concentrated by ammonium sulphate precipitation. Aliquots of $15 \mu \mathrm{g}$ protein were separated by SDS-PAGE on 15\% separating gels and transferred onto $0 \cdot 2 \mu \mathrm{M}$ polyvinylidene difluoride membranes (Osmonics, Westborough, MA, USA) by electroblotting. Membranes were incubated with a 1:3000 dilution of either the P450 scc or $5 \alpha$ RII antibody in Tris-buffered saline Tween, for $1 \mathrm{~h}$ at $22{ }^{\circ} \mathrm{C}$. The specificity of the P450 scc and $5 \alpha \mathrm{RII}$ antibodies has been reported previously (Nguyen et al. 2003a). The immune complexes were visualised by chemiluminescence and immunoblots were scanned and analysed using ImageQuaNT software (Amersham, Bucks, England). The densities of the bands were determined and were individually corrected for background by subtracting the density of the blank background area immediately below each band. The resulting value from each band was expressed in densitometric units.

\section{Neurosteroid RIA}

Neurosteroids were extracted from brain or adrenal tissue and plasma as described previously (Barbaccia et al. 1992, Nguyen et al. 2003a). Briefly, brain or adrenal gland tissue was homogenised three times with $50 \%$ methanol containing $1 \%$ acetic acid and the supernatants pooled. The supernatant was then applied to pre-primed Sep-Pak $\mathrm{C}_{18}$ cartridges (Waters Corp., Milford, MA, USA), steroids eluted with 100\% methanol, the collected fractions dried under nitrogen, and the steroids redissolved in $1.0 \mathrm{ml}$ assay buffer $(0 \cdot 1 \mathrm{M}$ PBS, pH 7.0). The recovery of steroids was monitored by adding trace amounts $(5000$ c.p.m.) of titrated standard to two homogenate samples that were run in parallel with each extraction run. The recovery was 80 $\pm 5 \%$ for allopregnanolone ( $n=4$ extractions), $75 \pm 5 \%$ for progesterone ( $n=4$ extractions) and $85 \% \pm 5 \%$ for pregnenolone ( $n=4$ extractions). Corrections for losses during extraction were included in final calculations.

Allopregnanolone was measured by specific radioimmunoassay, as described previously (Billiards et al. 2002, Nguyen et al. 2003a) using a polyclonal antibody purchased from Dr R H Purdy (San Diego, CA, USA). The antiserum displays low cross-reactivity with related steroids and the charactisation of the antiserum has been reported previously (Bernardi et al. 1998, Nguyen et al. 2003a). The limit of detection for allopregnanolone was $0.19 \pm 0.03 \mathrm{pmol} /$ tube $(n=4)$ and the intra- and interassay coefficients of variance were $5 \%$ and $10 \%$, respectively. Pregnenolone and progesterone were measured by specific radioimmunoassay using antibodies purchased from ICN Biomedicals (Seven Hills, NSW, Australia) and provided by Dr J Malecki (Bairnsdale, Victoria, Australia) respectively, as described previously (Rice et al. 1986). The cross-reactivity of the pregnenolone and progesterone antisera have been reported previously (Rice et al. 1986, Nguyen et al. 2003a). The limit of detection was $0 \cdot 10$ $\pm 0.02 \mathrm{pmol} /$ tube for pregnenolone $(n=4)$ and 0.12 $\pm 0.03 \mathrm{pmol} /$ tube for progesterone $(n=4)$. The intra- and interassay coefficients of variance were $5 \%$ and $15 \%$ respectively for pregnenolone, and $8 \%$ and $17 \%$ respectively for progesterone.

\section{irACTH RIA}

irACTH concentrations in plasma were measured without extraction, using a commercial kit (Diasorin Inc., Stillwater, Minnesota, USA) (Bocking \& Harding 1986). The intra-assay coefficient of variation was $6.0 \%$ and the sensitivity of the assay was $15 \mathrm{pg} / \mathrm{ml}$.

\section{Cortisol RIA}

Cortisol was extracted from plasma with dichloromethane and measured by radioimmunoassay as described previously (Bocking \& Harding 1986). The intra-assay coefficient was $8 \%$ and the sensitivity of the assay was $0 \cdot 40 \pm 0 \cdot 01 \mathrm{ng} / \mathrm{ml}$.

\section{Statistical analysis}

Data are shown as mean \pm S.E.M. All data were analysed using statistical software (SPSS Version 9.0, Chicago, IL, USA). Changes in plasma cortisol and irACTH, plasma steroid concentrations, adrenal gland steroid content and brain neurosteroid content were analysed by a twoway repeated measures ANOVA, followed by Student Newman-Keuls post hoc test. The significance of changes in brain neurosteroidogenic enzyme content was determined by Student's $t$-test, followed by a Holm's stepdown modification of Bonferroni's multiple comparisons procedure (Ludbrook 1998). Differences in adrenal gland neurosteroidogenic enzyme content were compared using Student's unpaired $t$-test. $P<0 \cdot 05$ was considered to be statistically significant.

\section{Results}

Plasma irACTH and steroid concentrations

After hypophysectomy, plasma irACTH concentrations decreased by $60-70 \%$ compared with those in sham 
Table 1 Fetal plasma ACTH and cortisol concentrations in control, hypophysectomised or adrenalectomised fetuses

\begin{tabular}{|c|c|c|c|}
\hline & Intact $(n=5) 1$ & $\mathbf{H X}(n=5)$ & ADX $(n=5)$ \\
\hline АСТН $(\mathrm{pg} / \mathrm{ml})$ & $31 \cdot 8 \pm 6 \cdot 0^{\mathrm{a}}$ & $10 \cdot 6 \pm 2 \cdot 2^{b}$ & $490 \cdot 3 \pm 149 \cdot 4^{\mathrm{c}}$ \\
\hline Cortisol (ng/ml) & $22 \cdot 7 \pm 7 \cdot 7^{\mathrm{a}}$ & $5 \cdot 0 \pm 0 \cdot 3^{b}$ & $4 \cdot 2 \pm 0 \cdot 4^{b}$ \\
\hline
\end{tabular}

Values shown are fetal ACTH and cortisol concentrations in plasma collected at postmortem (140 days of gestation) in intact,

hypophysectomised (HX) or adrenalectomised (ADX) fetuses. Data presented as means \pm S.E.M. Different lower case letters indicate significant differences between groups $(P<0 \cdot 05)$.

operated fetuses, and plasma cortisol was decreased from $22 \cdot 7$ to $5 \cdot 0 \mathrm{ng} / \mathrm{ml}$, a value not different from that present in the adrenalectomised fetuses (Table 1). After adrenalectomy, the very low concentrations of cortisol were associated with a significant (16-fold) increase in plasma irACTH (Table 1), consistent with findings of previous studies (Unno et al. 1998, Simmonds et al. 2001) and with a lack of negative feedback by cortisol on the anterior pituitary (Coulter et al. 1992).

Plasma concentrations of allopregnanolone were significantly and similarly reduced after hypophysectomy or adrenalectomy compared with those in intact fetuses ( $\sim 30 \%$ of intact values), with no difference between the hypophysectomised and adrenalectomised fetuses (Fig. 1A). Plasma progesterone and pregnenolone concentrations in adrenalectomised and hypophysectomised fetuses were also significantly less than those in intact fetuses ( $\sim 25 \%$ and $50 \%$ of intact values, respectively), with no significant difference between these two surgical groups (Fig. 1B and $\mathrm{C}$ respectively).

\section{Brain and adrenal gland steroid content}

Similar to findings in plasma, adrenal contents of allopregnanolone, progesterone and pregnenolone were markedly lower in the hypophysectomised fetuses than in intact fetuses at 140 days of gestation (Fig. 1A, B and C, respectively). In contrast to the findings in plasma and adrenal gland, allopregnanolone and progesterone content of the cerebellum, medulla and PMC were not significantly changed by either hypophysectomy or adrenalectomy (Fig. 1A and B, respectively). There was also no change in pregnenolone content in these regions, except for the medulla, in which pregnenolone was increased significantly after adrenalectomy (Fig. 1C). There was no difference between any of the groups with respect to content of allopregnanolone, progesterone or pregnenolone in the hippocampus or hypothalamus/ thalamus (data not shown).

\section{Brain and adrenal gland P450scc/5a-RII content}

The expressions of P450 scc and $5 \alpha$ RII were examined in the same tissues as were used for measurement of steroid

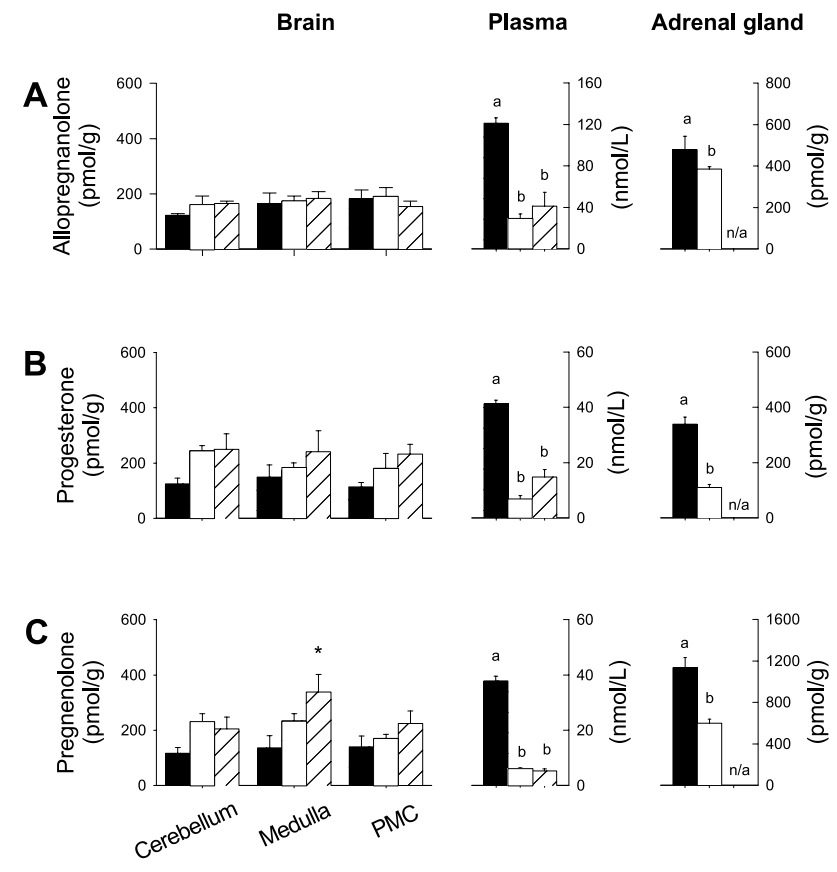

Figure 1 (A) Allopregnanolone, (B) progesterone and (C) pregnenolone concentrations in fetal brain tissue (cerebellum, medulla and PMC) and plasma and adrenal glands collected from intact fetuses $(n=5 ; \boldsymbol{\square})$, hypophysectomised fetuses $(n=6 ; \square)$ and adrenalectomised fetuses ( $n=6$; hatched bars) at 140 days of gestation. Note the differences in scale between concentrations in plasma and the adrenal gland. Each bar represents the mean \pm S.E.M. Different lower case letters indicate a significant difference between groups $(P<0 \cdot 05)$.

content. In the adrenal gland, P450 scc content was significantly decreased after hypophysectomy compared with that in intact fetuses (Fig. 2A), whereas $5 \alpha$ RII content was not changed (Fig. 2B). In the brain, P450 scc and $5 \alpha$ RII contents were not changed by either hypophysectomy or adrenalectomy, in any region examined (Fig. 2A and B).

\section{Discussion}

The principal finding of this study was that, whereas both adrenalectomy and hypophysectomy resulted in the reduction of plasma concentrations of the neurosteroid allopregnanolone, there were no changes in the concentrations of this steroid in any region of the fetal brain that we examined. Secondly, although expression of the rate-limiting steroidogenic enzyme, P450 scc (but not $5 \alpha \mathrm{RII})$, in the adrenal gland was decreased after hypophysectomy, the expression of neither P450 scc nor 5aRII was changed in the brain. These findings suggest that concentrations of allopregnanolone in the fetal brain are not significantly influenced by circulating irACTH and cortisol concentrations, or by adrenal steroidogenesis. 
Cerebellum

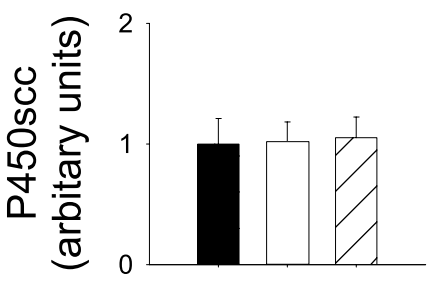

B

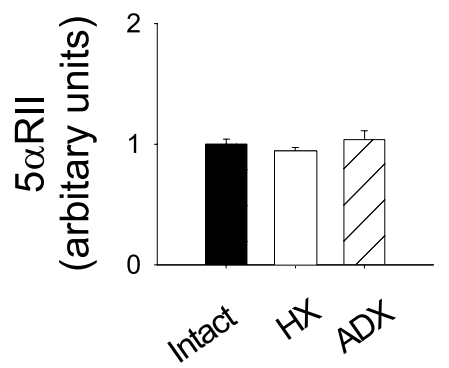

Medulla
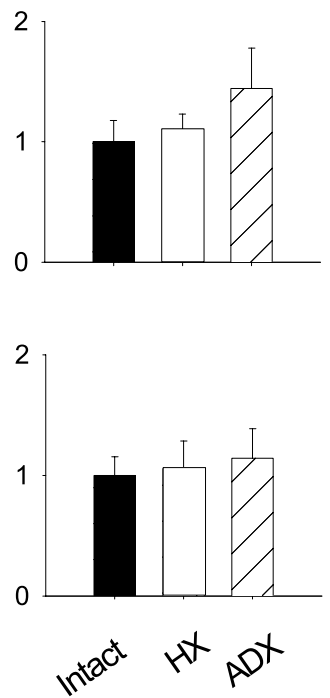

PMC
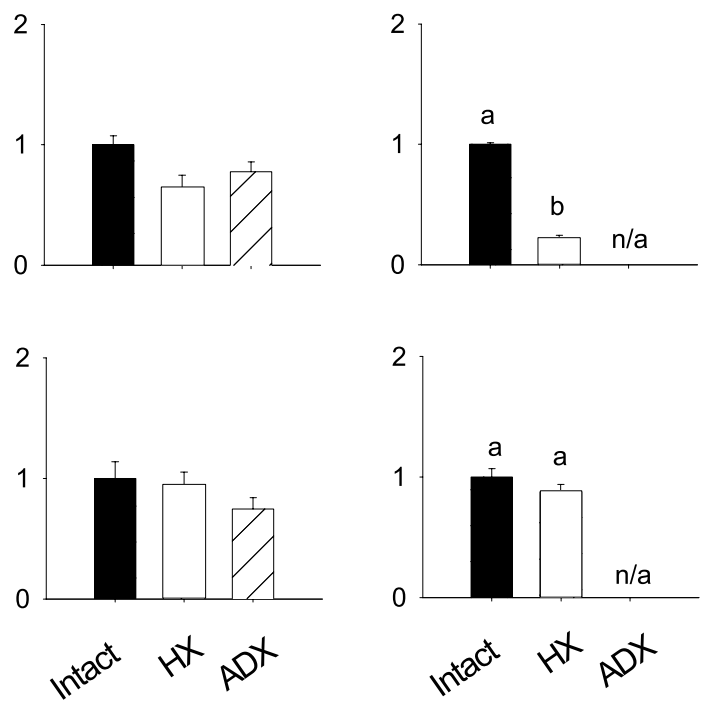

Adrenal gland

Figure 2 Expression of (A) P450 scc and (B) $5 \alpha$ RII in fetal brain tissue (cerebellum, medulla and PMC) and adrenal glands collected from intact fetuses $(n=5 ; \mathbf{\square})$, hypophysectomised fetuses $(n=6 ; \mathrm{HX}, \square)$ and adrenalectomised fetuses $(n=6$; ADX, hatched bars) at 140 days of gestation. Each bar represents the mean \pm S.E.M. Different lower case letters indicate a significant difference between groups $(P<0 \cdot 05)$.

Allopregnanolone may be synthesised de novo from cholesterol in the brain, but a proportion of this steroid may derive from precursors present in the circulation that are taken up by the brain (Miller 1987, Simmonds et al. 2001, Nguyen et al. 2003a). Thus peripheral steroidogenesis might influence neuroactive steroid content in the fetal brain. The finding that allopregnanolone, progesterone and pregnenolone concentrations in the fetal sheep brain did not decrease in any of the regions of the brain examined after either hypophysectomy or adrenalectomy indicates that brain steroid content is regulated independently of adrenal gland activity and, furthermore, does not depend on the plasma concentrations of these steroids.

An alternative explanation for the absence of a decline in allopregnanolone concentrations may be the presence of a novel active uptake mechanism that maintains brain concentrations in the presence of a decrease in plasma concentrations. However, to date no such mechanism has been identified. Our antiserum has a low cross-reactivity with related steroids, but we cannot exclude the possibility that it recognises unusual steroids or steroid-like molecules in the fetal brain that are not affected by the hypophysectomy and adrenalectomy procedures used in this study. However, such an explanation would require that such steroid-like molecules be present in the brain, and not in the circulation.

We found that hypophysectomy reduced plasma irACTH and cortisol concentrations, as expected. The decrease in irACTH was to an extent similar to that shown in previous studies and is consistent with total hypophysectomy, which typically causes a greater than 50\% decrease in irACTH (Phillips et al. 1996, Canny et al. 1998). Ectopic sources of irACTH remain after complete hypophysectomy; however, the irACTH from these sources is not steroidogenically active at the adrenal gland (Cudd \& Wood 1992). The decrease in cortisol concentrations reported in this study was also consistent with total hypophysectomy (Coulter et al. 1992) or adrenalectomy (Hennessy et al. 1982).

The finding that plasma allopregnanolone, pregnenolone and progesterone concentrations decreased to less than $25 \%$ of intact fetal values after either hypophysectomy or adrenalectomy indicates that adrenal synthesis is responsible for much of the circulating concentrations of these steroids in the fetal circulation. These observations are consistent with findings from studies performed in the adult rat (Corpechot et al. 1993). The plasma concentrations of these steroids in the intact fetuses were similar to those we have reported previously (Nicol et al. 1997, Nguyen et al. 2003a).

The present findings may also provide an explanation for the considerable residual concentrations of progesterone that remain after inhibition of placental progesterone synthesis (Crossley et al. 1997). The concentrations of these steroids remaining after hypophysectomy and adrenalectomy are likely to result from placental secretion, and possibly from placental transfer of maternally derived steroids. However, the low residual concentrations of allopregnanolone in the plasma suggest that maternal or placental sources contribute little to the concentrations of 
allopregnanolone in the fetal brain and are likely to have a limited role in regulating the allopregnanolone-induced stimulation of GABAergic activity in the fetus (Nicol et al. 1999). Although these findings indicate that the final enzymatic steps for producing allopregnanolone are performed in the tissues of the brain, they do not preclude the possibility that other steroids (for example 4-pregnene$20 \alpha$-ol-3-one and 4-pregnene-3 $\alpha$-ol-20-one) could be used by the fetal brain as precursors in the synthesis of allopregnanolone.

The present study also examined the expression of the two key rate-limiting steroidogenic enzymes responsible for the production of pregnenolone and allopregnanolone: P450 scc and $5 \alpha$ RII respectively. The finding that both enzymes were expressed strongly in the fetal brain and adrenal gland is consistent with our previous observations (Nguyen et al. 2003a, c) and studies in rodents by others (Voutilainen \& Miller 1986, Compagnone et al. 1995, Narasaka et al. 2001). Expression of P450 scc was significantly decreased in the adrenal gland after hypophysectomy, consistent with the stimulatory role of ACTH in the regulation of adrenal expression of $\mathrm{P} 450 \mathrm{scc}$ and production of pregnenolone in the fetus (Simmonds et al. 2001). ACTH may induce steroidogenesis in the adrenal gland via cAMP-stimulated production of pregnenolone after either chronic or acute stimulation. Chronic stimulation induces P450 scc gene transcription, leading to increased expression of $\mathrm{P} 450 \mathrm{scc}$ protein and increased steroidogenic capacity. Acute regulation occurs through the phosphorylation of pre-existing steroidogenic acute regulatory protein (StAR), in addition to synthesis of new StAR protein (Stocco 2000). An increase in StAR increases the flow of cholesterol into mitochondria, thus increasing availability to P450 scc.

In contrast to adrenal expression, P450 scc content in the fetal brain was unchanged after either hypophysectomy or adrenalectomy, suggesting that expression of $\mathrm{P} 450$ scc in the developing brain is regulated independently of the adrenal gland, and by mechanisms that do not involve either ACTH or cortisol. We have previously reported that expression of $\mathrm{P} 450 \mathrm{scc}$ is upregulated in the fetal brain by acute hypoxia (Nguyen et al. 2003b), an effect that may depend on increased expression of hypoxia-sensitive transcription factors (such as hypoxia-inducible factor-1 $\alpha$ ), rather than $\mathrm{ACTH}$. These factors may be responsible for the pregnenolone content in the medulla, which was increased after adrenalectomy, accompanied by a rise in P450 scc expression, which may be responsible for the increase in pregnenolone content. These findings suggest that ACTH-independent mechanisms such as an increase in the expression of hypoxia-inducible factors may be possible regulators of $\mathrm{P} 450 \mathrm{scc}$ in the fetal brain. The absence of altered $5 \alpha$ RII expression in either the adrenal gland or the brain after hypophysectomy or adrenalectomy suggests that the regulatory pathways responsible for controlling $5 \alpha \mathrm{RII}$ content are different from those controlling
P450 scc. Analysis of the 5aRII gene in the mouse brain showed the presence of a progesterone regulatory element (Matsui et al. 2002). However, the role of progesterone in the developing brain remains equivocal, as we found that the concentrations of progesterone decreased in plasma after hypophysectomy or adrenalectomy, but were maintained in the brain, and there was no change in $5 \alpha$ RII content. Glucocorticoid receptors are expressed in the fetal brain from mid-gestation, with concentrations peaking around 110-120 days of gestation, and are also functional at this time (Matthews et al. 1995, Dodic et al. 2002). These observations indicate that the absence of an effect of declining glucocorticoid concentration on enzyme expression does not result from the lack of expression of glucocorticoid receptor in the fetal brain.

We have previously shown that the concentrations of allopregnanolone in the fetal brain decrease rapidly after birth, suggesting a major role for placental factors or the intrauterine environment for maintaining concentrations of this neurosteroid in the fetal brain (Nguyen et al. 2003a). In previous studies, we also examined the effect of chronic hypoxaemia using a model of placental insufficiency and found that plasma progesterone concentrations were decreased and allopregnanolone concentrations increased under these hypoxic conditions. However, there were no significant changes in the brain content of these steroids (Nguyen et al. 2003c). The ability of the fetal brain to maintain high concentrations of allopregnanolone, progesterone and pregnenolone despite ablation of the pituitary or adrenal gland, and in the presence of placental impairment sufficient to severely retard fetal growth, supports the contention that steroidogenesis in the developing brain is not dependent on either adrenal or placental production of steroid. These observations are consistent with the notion that fetal brain does indeed have the independent capacity to maintain steroid synthesis during development and that this is responsible, at least in part, for the high concentrations of neurosteroids found in the fetal brain compared with that of the adult.

In summary, we have shown a marked decline in adrenal allopregnanolone content and plasma allopregnanolone concentration after both adrenalectomy and hypophysectomy, but no change within the fetal brain. Expression of P450 scc in the adrenal gland and plasma pregnenolone concentrations were reduced after hypophysectomy whereas, again, the brain was unaffected. These observations indicate that brain neurosteroid synthesis is regulated independently of the HPA axis, plasma allopregnanolone and cortisol concentrations. Thus mechanisms within the fetal brain appear to be of primary importance in the control of allopregnanolone concentrations.

\section{Acknowledgements}

The authors thank Alex Satragno for surgical assistance. This study was supported by a Monash University Faculty 
of Medicine Postgraduate Scholarship to P N N and a National Health and Medical Research Council of Australia Grant No. 143503 to J J H \& D W W.

\section{References}

Barbaccia ML, Roscetti G, Trabucchi M, Ambrosio C \& Massotti M 1992 Cyclic AMP-dependent increase of steroidogenesis in brain cortical minces. European Journal of Pharmacology 219 485-486.

Bernardi F, Salvestroni C, Casarosa E, Nappi RE, Lanzone A, Luisi S, Purdy RH, Petraglia F \& Genazzani AR 1998 Aging is associated with changes in allopregnanolone concentrations in brain, endocrine glands and serum in male rats. European Journal of Endocrinology 138 316-321.

Billiards SS, Walker DW, Canny BJ \& Hirst JJ 2002 Endotoxin increases sleep and brain allopregnanolone concentrations in newborn lambs. Pediatric Research 52 892-899.

Bocking AD \& Harding R 1986 Effects of reduced uterine blood flow on electrocortical activity, breathing, and skeletal muscle activity in fetal sheep. Amercian Journal of Obstetrics and Gynecology 154 655-662.

Canny BJ, Young IR \& Veldhuis JD 1998 Hypothalamo-pituitary disconnection of the late-gestation ovine fetus results in profound changes in cortisol secretion that are not reflected in commensurate changes in adrenocorticotropin secretion. Endocrinology 139 3210-3219.

Cheney DL, Uzunov D, Costa E \& Guidotti A 1995 Gas chromatographic-mass fragmentographic quantitation of 3 alpha-hydroxy-5 alpha-pregnan-20-one (allopregnanolone) and its precursors in blood and brain of adrenalectomized and castrated rats. Journal of Neuroscience 15 4641-4650.

Compagnone NA, Bulfone A, Rubenstein JL \& Mellon SH 1995 Steroidogenic enzyme P450c17 is expressed in the embryonic central nervous system. Endocrinology 136 5212-5223.

Corpechot C, Young J, Calvel M, Wehrey C, Veltz JN, Touyer G, Mouren M, Prasad VV, Banner C, Sjovall J et al. 1993

Neurosteroids: 3 alpha-hydroxy-5 alpha-pregnan-20-one and its precursors in the brain, plasma, and steroidogenic glands of male and female rats. Endocrinology 133 1003-1009.

Coulter CL, Young IR, Browne CA \& McMillen IC 1992 The effect of fetal hypophysectomy with or without ACTH replacement on the molecular weight profile of enkephalin-containing peptides in the adrenal medulla of the fetal sheep. Journal of Endocrinology 134 369-375.

Crawley JN, Glowa JR, Majewska MD \& Paul SM 1986 Anxiolytic activity of an endogenous adrenal steroid. Brain Research $\mathbf{3 9 8}$ 382-385.

Crossley KJ, Nicol MB, Hirst JJ, Walker DW \& Thorburn GD 1997 Suppression of arousal by progesterone in fetal sheep. Reproduction, Fertility and Development 9 767-773.

Crossley KJ, Walker DW, Beart PM \& Hirst JJ 2000 Characterisation of $\mathrm{GABA}(\mathrm{A})$ receptors in fetal, neonatal and adult ovine brain: region and age related changes and the effects of allopregnanolone. Neuropharmacology 39 1514-1522.

Cudd TA \& Wood CE 1992 Prostaglandin E2 releases ovine fetal ACTH from a site not perfused by the carotid vasculature. Amercian Journal of Physiology 263 R136-R140.

Dodic M, Hantzis V, Duncan J, Rees S, Koukoulas I, Johnson K, Wintour EM \& Moritz K 2002 Programming effects of short prenatal exposure to cortisol. FASEB Journal 16 1017-1026.

Hennessy DP, Coghlan JP, Hardy KJ \& Wintour EM 1982 Development of the pituitary-adrenal axis in chronically cannulated ovine fetuses. Journal of Developmental Physiology 4 339-352.

Ludbrook J 1998 Multiple comparison procedures updated. Clinical and Experimental Pharmacology and Physiology 25 1032-1037.
Matsui D, Sakari M, Sato T, Murayama A, Takada I, Kim M, Takeyama K \& Kato S 2002 Transcriptional regulation of the mouse steroid 5 alpha-reductase type II gene by progesterone in brain. Nucleic Acids Research 30 1387-1393.

Matthews SG, Yang K \& Challis JR 1995 Changes in glucocorticoid receptor mRNA in the developing ovine pituitary and the effects of exogenous cortisol. Journal of Endocrinology 144 483-490.

Mesiano S, Young IR, Baxter RC, Hintz RL, Browne CA \& Thorburn GD 1987 Effect of hypophysectomy with and without thyroxine replacement on growth and circulating concentrations of insulin-like growth factors I and II in the fetal lamb. Endocrinology 120 1821-1830.

Miller WL 1987 Structure of genes encoding steroidogenic enzymes. Journal of Steroid Biochemistry 27 759-766.

Narasaka T, Suzuki T, Moriya T \& Sasano H 2001 Temporal and spatial distribution of corticosteroidogenic enzymes immunoreactivity in developing human adrenal. Molecular and Cellular Endocrinology 174 111-120.

Nguyen PN, Billiards SS, Walker DW \& Hirst JJ 2003a Changes in 5 alpha-pregnane steroids and neurosteroidogenic enzyme expression in the perinatal sheep. Pediatric Research 53 956-964.

Nguyen PN, Yan EB, Scott KM, Castillo-Melendez M, Walker DW \& Hirst JJ $2003 b$ Increased allopregnanolone concentrations in the fetal brain following umbilical cord occlusion. Journal of the Society for Gynecologic Investigation 10 187A (Abstract No. 294).

Nguyen PN, Billiards SS, Walker DW \& Hirst JJ 2003c Changes in 5 alpha-pregnane steroids and neurosteroidogenic enzyme expression in fetal sheep with umbilicoplacental embolisation. Pediatric Research 54 840-847.

Nicol MB, Hirst JJ, Walker DW \& Thorburn GD 1997 Effect of alteration of maternal plasma progesterone concentrations on fetal behavioural state during late gestation. Journal of Endocrinology 152 379-386.

Nicol MB, Hirst JJ \& Walker DW 1998 Effect of pregnane steroids on electrocortical activity and somatosensory evoked potentials in fetal sheep. Neuroscience Letters 253 111-114.

Nicol MB, Hirst JJ \& Walker DW 1999 Effects of pregnanolone on behavioural parameters and the responses to GABA(A) receptor antagonists in the late gestation fetal sheep. Neuropharmacology $\mathbf{3 8}$ 49-63.

Nicol MB, Hirst JJ \& Walker DW 2001 Effect of finasteride on behavioural arousal and somatosensory evoked potentials in fetal sheep. Neuroscience Letters 306 13-16.

Phillips ID, Ross JT, Owens JA, Young IR \& McMillen IC 1996 The peptide ACTH(1-39), adrenal growth and steroidogenesis in the sheep fetus after disconnection of the hypothalamus and pituitary. Journal of Physiology 491 871-879.

Pomata PE, Colman-Lerner AA, Baranao JL \& Fiszman ML 2000 In vivo evidences of early neurosteroid synthesis in the developing rat central nervous system and placenta. Brain Research. Developmental Brain Research 120 83-86.

Purdy RH, Morrow AL, Moore PH Jr \& Paul SM 1991 Stress-induced elevations of gamma-aminobutyric acid type A receptor-active steroids in the rat brain. PNAS $\mathbf{8 8} 4553-4557$.

Rice GE, Jenkin G \& Thorburn GD 1986 Comparison of particle-associated progesterone and oxytocin in the ovine corpus luteum. Journal of Endocrinology 108 109-116.

Simmonds PJ, Phillips ID, Poore KR, Coghill ID, Young IR \& Canny BJ 2001 The role of the pituitary gland and ACTH in the regulation of mRNAs encoding proteins essential for adrenal steroidogenesis in the late-gestation ovine fetus. Journal of Endocrinology 168 475-485.

Simonetta G, Young IR, Coulter CL, Hey NJ \& McMillen IC 1993 Fetal adrenalectomy does not affect circulating enkephalins in the sheep fetus during late gestation. Neuroendocrinology $\mathbf{5 7} 408-415$.

Stocco DM 2000 Intramitochondrial cholesterol transfer. Biochimica et Biophysica Acta 1486 184-197. 
Unno N, Wu WX, Ding XY, Li C, Hing WK \& Nathanielsz PW 1998 The effects of fetal adrenalectomy at 110 days gestational age on AVP and CRH mRNA expression in the hypothalamic paraventricular nucleus of the ovine fetus. Brain Research. Developmental Brain Research 106 119-128.

Voutilainen R \& Miller WL 1986 Developmental expression of genes for the stereoidogenic enzymes P450 scc (20,22-desmolase),
P450c17 (17 alpha-hydroxylase/17,20-lyase), and P450c21 (21-hydroxylase) in the human fetus. Journal of Clinical Endocrinology and Metabolism 63 1145-1150.

Received 17 March 2004

Accepted 14 April 2004 